\title{
Editorial: Adaptive Kinetic-Fluid Models for Plasma Simulations on Modern Computer Systems
}

\author{
Vladimir Kolobov ${ }^{1,2 *}$ and Fabrice Deluzet ${ }^{3}$ \\ ${ }^{1}$ CFD Research Corporation, Huntsville, AL, United States, ${ }^{2}$ The Center for Space Plasma and Aeronomic Research, The \\ University of Alabama in Huntsville, Huntsville, AL, United States, ${ }^{3}$ Institut de Mathématiques de Toulouse, Universitê de \\ Toulouse, CNRS, IMT, Université Paul Sabatier, Toulouse, France
}

Keywords: Boltzmann, Vlasov, Fokker-Planck kinetic equations, discrete velocity method, Particle-In-Cell methods, collisionless magnetized plasma, gas discharges, solar wind

\section{Editorial on the Research Topic}

\section{Adaptive Kinetic-Fluid Models for Plasma Simulations on Modern Computer Systems}

Most plasmas are highly non-equilibrium systems, and are characterized by a variety of disparate temporal and spatial scales. They must be described at the kinetic level in term of velocity distribution functions for electrons, ions, and neutral species. Under certain circumstances, simpler hydrodynamic (fluid) descriptions can be applied. Deep understanding of particle kinetics is required for identifying the applicability of fluid description and selecting appropriate closure models, which depend on plasma conditions (collisionless vs. collisional, magnetized vs. nonmagnetized), the particle type and energy. The challenges of plasma simulations can be addressed by dedicated multiscale numerical methods such as Implicit-Explicit (ImEx) methods, AsymptoticPreserving schemes, and hybrid methods [1-3].

This topic is devoted to adaptive kinetic-fluid models for plasma simulations, and contains eight original contributions illustrating the state-of-the-art.

Pfau-Kempf et al. describe Vlasiator-a numerical solver for collisionless magnetized plasma based on direct numerical solution of the Vlasov kinetic equation using Discrete Velocity Models (DVM). The solver is specifically designed for studies of solar wind interactions with near-Earth environment including the bow shock and magnetosheath regions. It is shown that the spatial and velocity resolutions should be adapted together rather than independently for optimal efficiency. This contributes to the ongoing discussion [4-7], of splitting vs non-splitting methods for mesh adaptation in phase space.

Lautenbach and Grauer describe a hierarchical treatment of collisionless magnetized plasmas combining the Vlasov treatment for ions and electrons at the fine level with a 10-moment fluid model at the coarse level for the Geospace Environmental Modeling of magnetic reconnection. We wish to add that new closures for the kinetic-fluid simulations of collisionless plasma have been recently proposed in Hunana et al.[8].

Markidis et al. introduce a Particle-In-Cell framework to cope with the dynamic coupling of kinetic and fluid plasma modeling. The PolyPic method makes use of computational particles for the discretization of both fluid and kinetic equations with the ability for fluid particles to become kinetic when required. This new approach offers promising gains in term of computational efficiency since only a small number of numerical particles is sufficient to represent fluid quantities. This provides an alternative approach to Micro-Macro Particle methods $[9,10]$.

Dynamically Adaptive Mesh Refinement (AMR) is an important tool to address the nonlinear nature of plasmas and increase accuracy and efficiency of simulations. Fujimoto uses AMRPIC model for efficient kinetic simulation of magnetic reconnection using dynamically adaptive Cartesian mesh. 
The challenges of vastly different time scales due to the disparity of electron and ion mass can be addressed with implicit schemes and coupled algorithms for particle transport and electromagnetics. Ho et al. present adaptive plasma simulations coupling multi-fluid models with a Vlasov-Maxwell and an MHD model. The issues related to the disparate time scales and the collision processes in PIC codes are discussed in Gueroult et al..

Roytershteyn and Delzanno introduce a new spectral method [11] to alleviate the numerical cost of kinetic plasma description together with a parallelization on distributed memory architectures. It relies on an approximation of the distribution function into Fourier modes in the physical space while the velocity space is sampled with Hermite modes. By adjusting the number of Hermite polynomials, it is possible to operate a smooth transition between fluid and kinetic plasma descriptions. The method is shown to be accurate with few Hermite modes in the linear theory, while non-linear plasma turbulence, characteristic of solar winds, is successfully reproduced with a refined sampling of the velocity space.

Another issue emphasizing the multiscale nature of plasma physics is related to plasma quasi-neutrality. The Debye length is routinely very small compared to plasma size and the characteristic time scale of interest is large compared to the reciprocal plasma frequency. It is therefore seducing to embed the quasi-neutrality assumption into plasma simulations, in order

\section{REFERENCES}

1. Dimarco G, Pareschi L. Numerical methods for kinetic equations. Acta Numer. (2014) 23:369-520. doi: 10.1017/S09624929140 00063

2. Degond P, Deluzet F. Asymptotic-preserving methods and multiscale models for plasma physics. J Comput Phys. (2017) 336:429-57. doi: 10.1016/j.jcp.2017.02.009

3. Hu J, Jin S, Li Q. Chapter 5 - Asymptotic-Preserving Schemes for Multiscale Hyperbolic and Kinetic Equations. In: Abgrall R, Shu C.-W, editors. Handbook of Numerical Analysis, Vol. 18, Supplement C Vols. Elsevier (2017). p. 103-29.

4. Kolobov V, Arslanbekov R, Levko D. Boltzmann-Fokker-Planck Kinetic Solver with Adaptive Mesh in Phase Space. In: AIP Conference Proceedings. Available online at: https://arxiv.org/abs/1810.09049 (2019)

5. Deriaz E, Peirani S. Six-dimensional adaptive simulation of the Vlasov equations using a hierarchical basis. Multiscale Model Simul. (2018) 16:583614. doi: 10.1137/16M1108649

6. Tanaka S, Yoshikawa K, Minoshima K, Yoshida N. Multidimensional Vlasov-Poisson simulations with high-order monotonicity- and positivitypreserving schemes. Astrophys J. (2017) 849:76 doi: 10.3847/1538-4357/ aa901f

7. Arslanbekov RR, Kolobov VI, Frolova AA. Kinetic solvers with adaptive mesh in phase space. Phys Rev E. (2013) 88:063301. doi: 10.1103/PhysRevE.88.063301

8. Hunana P, Tenerani A, Zank GP, Goldstein ML, Webb GM, Velli M, et al. A brief guide to fluid models with anisotropic temperatures Part 2 - Kinetic theory, Padé approximants and Landau fluid closures. Plasma Phys J. (2018) arXiv[Preprint].arXiv:1901.09360. Available online at: https://arxiv.org/abs/ 1901.09360 (accessed January 27, 2019). to filter out the fast/short scales from the model. Recently, numerical methods have been designed coupling quasi-neutral and non-quasi-neutral plasma models [12, 13]. A different route is explored in Arcese et al. who introduce an innovative method using a patch technique to dissociate the low-frequency plasma equations from the high frequency Maxwell system for electromagnetic fields. This numerical method reveals to be very efficient for simulations of microwave gas breakdown at atmospheric pressure.

\section{AUTHOR CONTRIBUTIONS}

All authors listed have made a substantial, direct and intellectual contribution to the work, and approved it for publication.

\section{ACKNOWLEDGMENTS}

VK was partially supported by the US Department of Energy Office of Fusion Energy Science Contract DE-SC0001939 and by the NSF EPSCoR project OIA-1655280 "Connecting the Plasma Universe to Plasma Technology in AL: The Science and Technology of Low-Temperature Plasma". FD was partially supported by the Fédération de Fusion pour la Fusion par Confinement Magnétique in the frame of the BRIDIPIC project «BRIDging Particle-In-Cell methods and low frequency numerical models of plasmas».

9. Crestetto A, Crouseilles N, Lemou M. Kinetic/fluid micro-macro numerical schemes for Vlasov-Poisson-BGK equation using particles. Kinetic Relat Models. (2012) 5:787-16. doi: 10.3934/krm.201 2.5.787

10. De Cecco A, Deluzet F, Negulescu C, Possanner S. Asymptotic transition from kinetic to adiabatic electrons along magnetic field lines. Multiscale Model. Simul. (2017) 15:309-38. doi: 10.1137/15M10 43686

11. Wang Y, Zhang S. Solving Vlasov-Poisson-Fokker-Planck equations using NRxx method. Commun Comput Phys. (2017) 21:782-807. doi: 10.4208/cicp.220415.080816a

12. Degond P, Deluzet F, Savelief D. Numerical approximation of the EulerMaxwell model in the quasineutral limit. J Comput Phys. (2012) 23:1917-46. doi: 10.1016/j.jcp.2011.11.011

13. Crouseilles N, Dimarco G, Vignal M. Multiscale schemes for the BGKVlasov-Poisson system in the quasi-neutral and fluid limits. Stability analysis and first order schemes. Multiscale Model. Simul. (2016) 65-95. doi: $10.1137 / 140991558$

Conflict of Interest Statement: The authors declare that the research was conducted in the absence of any commercial or financial relationships that could be construed as a potential conflict of interest.

Copyright (c) 2019 Kolobov and Deluzet. This is an open-access article distributed under the terms of the Creative Commons Attribution License (CC BY). The use, distribution or reproduction in other forums is permitted, provided the original author(s) and the copyright owner(s) are credited and that the original publication in this journal is cited, in accordance with accepted academic practice. No use, distribution or reproduction is permitted which does not comply with these terms. 